\title{
Direct Observation of Antiferromagnetic Parity Violation in the Electronic Structure of Mn2Au
}

\section{Olena Fedchenko}

Johannes Gutenberg-Universität Mainz

Libor Šmejkal

Johannes Gutenberg-University Mainz https://orcid.org/0000-0003-1193-1372

\section{Michael Kallmayer}

Johannes Gutenberg-Universität Mainz

\section{Yaryna Lytvynenko}

Johannes Gutenberg-Universität Mainz https://orcid.org/0000-0002-5607-8216

Katherina Medjanik

Johannes Gutenberg-Universität Mainz

Dmitry Vasilyev

Johannes Gutenberg-Universität Mainz

\section{Sergey Babenkov}

Johannes Gutenberg-Universität Mainz

\section{Jure Demsar}

Johannes Gutenberg University Mainz https://orcid.org/0000-0003-4551-7444

Mathias Kläui

Johannes Gutenberg University of Mainz https://orcid.org/0000-0002-4848-2569

Gerd Schönhense

Universität Mainz https://orcid.org/0000-0002-8921-2901

Martin Jourdan

Johannes Gutenberg-Universität Mainz https://orcid.org/0000-0001-6785-0518

Jairo Sinova

Johannes Gutenberg University

Hans-Joachim Elmers ( $\nabla$ elmers@uni-mainz.de)

Johannes Gutenberg-Universität Mainz https://orcid.org/0000-0002-2525-9954

\section{Article}

Keywords:

Posted Date: January 17th, 2022 
DOI: https://doi.org/10.21203/rs.3.rs-1010314/v1

License: (a) (i) This work is licensed under a Creative Commons Attribution 4.0 International License. Read Full License 


\title{
Direct Observation of Antiferromagnetic Parity Violation in the Electronic Structure of $\mathrm{Mn}_{2} \mathrm{Au}$
}

\author{
O. Fedchenko, ${ }^{1}$ L. Šmejkal,,${ }^{1,2}$ M. Kallmayer ${ }^{3}$ Ya. Lytvynenko, ${ }^{1}$ K. Medjanik, ${ }^{1}$ S. Babenkov, ${ }^{1}$ D. \\ Vasilyev, ${ }^{1}$ M. Kläui, ${ }^{1}$ J. Demsar, ${ }^{1}$ G. Schönhense, ${ }^{1}$ M. Jourdan, ${ }^{1}$ J. Sinova, ${ }^{1,2}$ and H. J. Elmers ${ }^{1}$ \\ ${ }^{1}$ Institut für Physik, Johannes Gutenberg-Universität Mainz, Staudingerweg 7, D-55099 Mainz, Germany \\ ${ }^{2}$ Inst. of Physics Academy of Sciences of the Czech Republic, Cukrovarnická 10, Praha 6, Czech Republic \\ ${ }^{3}$ Surface Concept GmbH, Am Sägewerk 23A, D-55124 Mainz, Germany
}

Parity symmetric photoemission spectra are ubiquitous in solid state research, being prevalent in many highly active areas, such as unconventional superconductors, nonmagnetic and antiferromagnetic topological insulators, and weakly relativistic collinear magnets, among others $[1,2]$. The direct observation of parity-violating[3-7] metallic Kramers degenerate bands has remained hitherto experimentally elusive. Here we observe the antiferromagnetic parity violation (APV) in the bandstructure of $\mathrm{Mn}_{2} \mathrm{Au}$ thin films by using momentum microscopy with sub- $\mu$ m spatial resolution, allowing momentum resolved photoemission on single antiferromagnetic domains. The APV arises from breaking the $\mathcal{P}$ symmetry of the underlying crystal structure by the collinear antiferromagnetism, while preserving the joint spacetime inverison $\mathcal{P} \mathcal{T}$-symmetry and in combination with large spin-orbit coupling $[3,6]$. In addition, our work also demonstrates a novel tool to directly image the Néel vector direction by combining spatially resolved momentum microscopy with ab-initio calculations.

Solid-state textbooks and angular-resolved photoemission spectroscopy (ARPES) studies commonly present energy bands that are parity symmetric in momentum, i.e. $E(k)=E(-k)$. This is enforced by the symmetries of the materials, such as inversion/parity $\mathcal{P}$, timereversal $\mathcal{T}$, time-reversal coupled with translation $\mathcal{T} \boldsymbol{t}$ or time-reversal coupled with the spin rotational symmetry $\mathcal{T} \mathcal{R}_{S}$, where $\mathcal{R}_{S}$ rotates the spin by $180^{\circ}$. Even, the Rashba materials (and noncentrosymmetric spin-orbit coupled systems in general), which break parity only in spin space, do preserve parity due to the $\mathcal{T}$ or $\mathcal{T} \boldsymbol{t}$ symmetry, i.e. $E^{\uparrow}(k)=E^{\downarrow}(-k)$. Therefore, from this perspective, bulk band structures violating parity in systems with Kramer degenerate bands are rather unique and rare. Bands with broken parity have appeared only in systems that break Kramers degeneracy by both spatial inversion and time symmetry at interfaces [8], and have been predicted to appear in complex noncoplanar magnets that break $\mathcal{T} R_{S}$ [4].

More recently, APV, i.e. $E(k) \neq E(-k)$, was predicted to arise in spin-orbit coupled collinear antiferromagnets such as CuMnAs and $\mathrm{Mn}_{2} \mathrm{Au}$, which break $\mathcal{P}$ and $\mathcal{T}$ symmetries but preserve its $\mathcal{P} \mathcal{T}$ combination, as
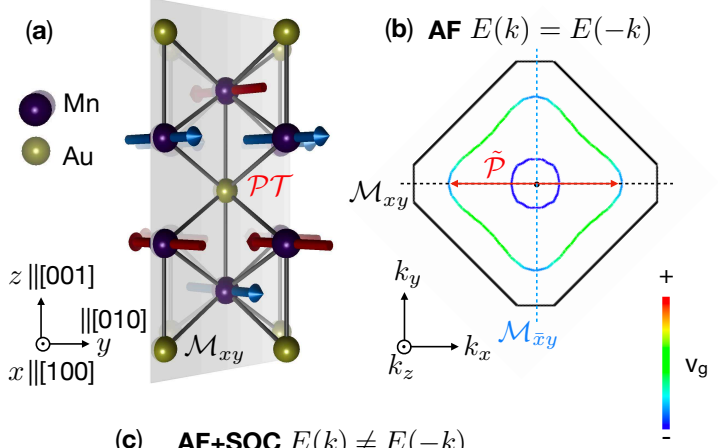

(c) $\mathbf{A F}+\mathbf{S O C} E(k) \neq E(-k)$

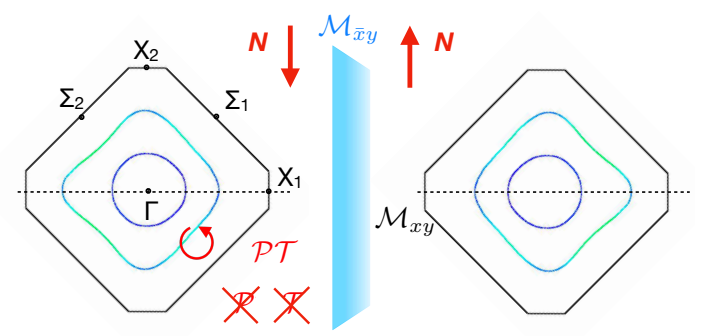

FIG. 1. (a) Structure of $\mathrm{Mn}_{2} \mathrm{Au}$ demonstrating the $\mathcal{P} \mathcal{T}$ symmetry in the magnetically ordered phase; (b) Calculated constant energy surface in momentum space at $-0.4 \mathrm{eV}$ of $\mathrm{Mn}_{2} \mathrm{Au}$ without SOC. The spin-group symmetry $\mathcal{R}_{S} \mathcal{T}$ in the decoupled spin and space sector preserve the bandstructure parity, acting as $\mathcal{P}$ in the band structure, denoted by $\tilde{\mathcal{P}}$. Color code denotes the group velocity $v_{g}$. (c) Asymmetric constant energy surface at energy $-0.4 \mathrm{eV}$ arising from the Néel order and SOC, shown for two directions of the Neél order connected by the mirror symmetries $\mathcal{M}_{x y}$ and $\mathcal{M}_{\bar{x} y}$.

we show in Fig. 1(a), in spite of having crystal parity, i.e., the crystal is itself centrosymmetric but the antiferromagnetic order breaks that symmetry. Without spinorbit coupling (SOC), the bandstructure is still symmetric [Fig. 1(b)], and its nonrelativistic symmetry group $P 1_{4} / 2_{m} 1_{m} 1_{m}[2,6]$ exhibits also the two mirror planes $\mathcal{M}_{x y}$ and $\mathcal{M}_{\bar{x} y}$ marked with dotted lines in black and blue color, respectively. With SOC, the magnetic symmetry group is $F m^{\prime} m m[6]$ and breaks the $\mathcal{M}_{\bar{x} y}$ symmetry. The bandstructure cannot be superposed with its parity image related by the blue mirror plane $\mathcal{M}_{\bar{x} y}$, as we illustrate in Fig. 1(c), exhibiting directly APV. Here $\mathcal{P} \mathcal{T}$ maps a state to its equal momentum state (with opposite spin) since the presence of spin-orbit coupling prevents the coupling to states with opposite spin and momenta, hence preserving Kramers degeneracy in these 
systems. Up to now, this predicted APV was observed only indirectly through the electrical current induced manipulation of the Néel vector via Néel spin-orbit torques (NSOT) in CuMnAs [9, 10] and $\mathrm{Mn}_{2} \mathrm{Au}[11]$, and secondorder magnetoresistance in CuMnAs [12].

The direct observation of APV using conventional ARPES is not possible due to averaging over many antiferromagnetic domains with typical sizes in the $\mu \mathrm{m}$ range. While a ferromagnetic capping layer can be used to orient the Néel vector via exchange coupling [13], the capping layer prevents surface sensitive ARPES measurements.

In this Letter, we use instead time-of-flight momentum microscopy combined with sub- $\mu \mathrm{m}$ spatial resolution (sub- $\mu$-ToFMM) to directly observe the APV by an asymmetric photoemission intensity, $E(k) \neq E(-k)$. Such a spatial resolution allows one to measure the electronic structure in areas restricted to single antiferromagnetic domains with well defined Néel vector orientation. We also further demonstrate below the direct imaging of the Néel vector direction, $\mathbf{N}$.

Epitaxial $\mathrm{Mn}_{2} \mathrm{Au}(001)$ films with a thickness of $40 \mathrm{~nm}$ were grown by rf-sputtering on $\mathrm{Al}_{2} \mathrm{O}_{3}(1 \overline{102})$ substrates with a $\mathrm{Ta}(001)$ buffer. $\mathrm{Mn}_{2} \mathrm{Au}$ has a body centered tetragonal crystal structure $\left(\right.$ bct $\left._{2}\right)$, with the (001) plane exhibiting a 4-fold structural symmetry and space group $I 4 / \mathrm{mmm}$. Details of the sample growth, characterization by X-ray and electron diffraction, as well as by atomic force microscopy, are reported in Refs. [14, 15]. During growth, the magnetic in-plane anisotropy aligns the Néel vector nearly equally distributed along both magnetic $\langle 110\rangle$ easy axes with domain sizes in the micrometer range [16]. Details of the sample, ARPES, ToFMM and photoemission measurements are given in the Supplementary Material.

In the experiment we first identify antiferromagnetic domains in real space by magnetic linear dichroism (MLD) in the photoemission electron microscopy (PEEM) mode. We then select single domain areas by the field aperture. Lastly, we record the photoemission intensity in momentum space by setting the electron optics to momentum mode.

The ToFMM with the electron optics set to the PEEM mode detects the spatial distribution of photoemission intensity, where the sample surface is magnified on the detector with a field-of-view of $50 \mu \mathrm{m}$ in diameter [Fig. 2(a)]. The spatial resolution in this mode is limited by the spherical aberration of the objective lens that increases with increasing parallel momentum of the detected electrons. The time-of-flight detection mode allows in addition to measure the kinetic energy of the photo-emitted electrons, hence simultaneously acquiring the three-dimensional data array $I\left(E_{B}, x, y\right)$.

In order to extract the magnetic contrast from the images, we exploit MLD. For reflected light, MLD originates from a magnetic-linear birefringence being sensitive to the magnetic order axis instead of its direction $[17,18]$.
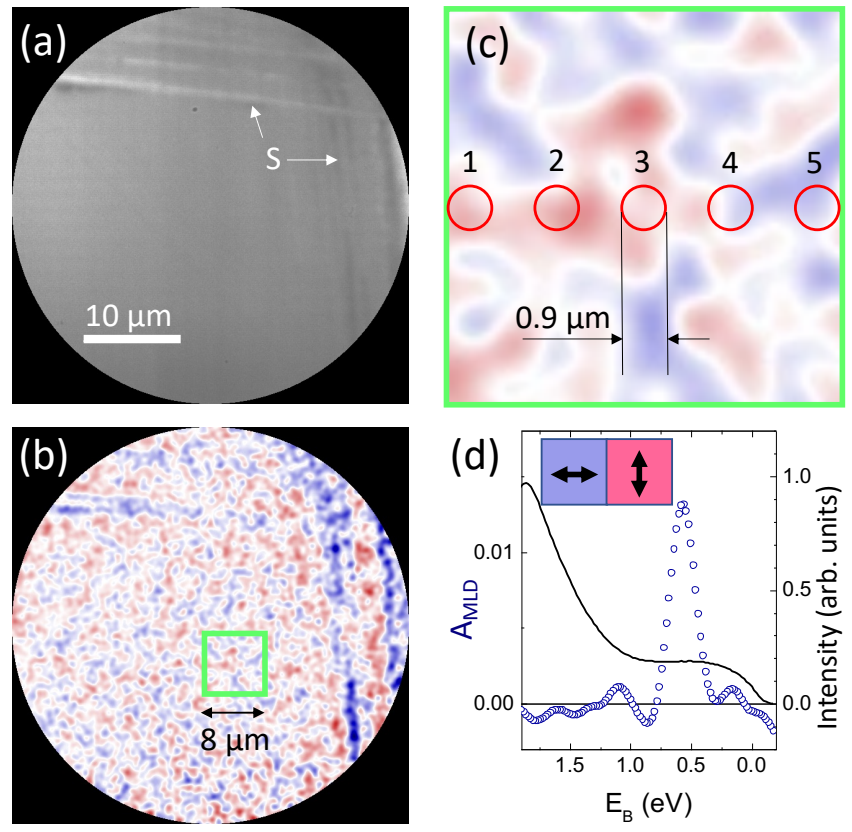

FIG. 2. (a) PEEM image of the $\mathrm{Mn}_{2} \mathrm{Au}(100)$ sample surface obtained with $6.4 \mathrm{eV}$ photon energy. Scratches (S) on the otherwise homogeneous surface serve for position determination. (b) Magnetic linear dichroism (MLD) image for the area as in (a) with color (red/blue) coded asymmetry $A_{\mathrm{MLD}}=\left(I_{p}-I_{s}\right) /\left(I_{p}+I_{s}\right)\left(E_{B}=0.6 \mathrm{eV}, p\right.$ - and $s$-polarized light). (c) Magnified image from the green square indicated in (b). The five numbered circles define the regions of interest selected by the field aperture that are used for momentum microscopy [results shown in Fig. 3(g-1 to g-5)]. (d) Intensity (black line) and MLD asymmetry, $A_{\mathrm{MLD}}$, (circles) vs. $E_{B}$.

For this reason, MLD can only distinguish between antiferromagnetic domains with perpendicular orientation. In the case of near threshold excitation of photoelectrons, related magnetic linear and circular dichroism effects have been observed [19-22]. In photoemission spectroscopy a similar effect can be observed [23]. X-ray magnetic linear dichroism photoemission electron microscopy (XMLD-PEEM) was successfully used to observe AFM domains in a wide range of materials [23-27], including both CuMnAs [10, 28] and $\mathrm{Mn}_{2} \mathrm{Au}$ thin films [16, 29].

We perform MLD photoemission microscopy by acquiring two data sets, exciting with linearly polarized laser light $(6.4 \mathrm{eV})$ parallel $(\mathrm{p})$ and perpendicular $(\mathrm{s})$ to the reflection plane of the laser beam.

The photoemission intensities for both measurements, integrated over the field of view, have first been normalized to each other. Then we calculate the spatial distribution of the MLD asymmetry $A_{\mathrm{MLD}}=\left[I_{p}\left(E_{B}, x, y\right)-\right.$ $\left.I_{s}\left(E_{B}, x, y\right)\right] /\left[I_{p}\left(E_{B}, x, y\right)+I_{s}\left(E_{B}, x, y\right)\right]$. Other contributions to the contrast, such as work function contrast, topographical contrast, impurities, and detector function are largely eliminated. Figs. 2(b,c) show the resulting magnetic contrast obtained at a binding energy $\left(E_{B}\right)$ of $0.6 \mathrm{eV}$. The color code for the corresponding Néel vector 
alignment is indicated as an inset in Fig. 2(d).

The result shows that it is indeed possible in this mode to distinguish domains with $\mathbf{N}$ parallel or perpendicular to $x$, but not whether $\mathbf{N}$ is pointing up or down [16].

We note that $A_{\mathrm{MLD}}$ depends on $E_{B}$. Fig. 2(d) depicts $A_{\mathrm{MLD}}\left(E_{B}\right)$ extracted from a single domain area in Fig. 2(c), revealing a pronounced asymmetry peak near $E_{B}=0.6 \mathrm{eV}$. We attribute the peak to a resonant excitation of a spin-orbit split state at this $E_{B}[6]$.

We next examine the results of photoemission in momentum space, depicted in Fig. 3. Assuming direct transitions into quasi-free-electron-like final states [30, 31], the final state momentum magnitude is given by:

$$
k_{\text {final }}=(1 / \hbar) \sqrt{2 m_{\text {eff }} E_{\text {final }}} ; \quad E_{\text {final }}=h \nu-E_{B}+V_{0}^{*},
$$

where $m_{\text {eff }}$ denotes the effective electron mass, $h \nu$ the photon energy, and $V_{0}^{*} \approx 10 \mathrm{eV}$ the inner potential with respect to the Fermi energy.

Figs. 3(a,b) show results obtained for excitation with $21.2 \mathrm{eV}$ photons using a hemispherical analyzer (see results for $E_{B}=0$ in Ref. 6 for comparison). In this case, the maximum value of the perpendicular momentum component is $k_{z}=2.86 \AA^{-1}=1.9 G_{001}$. The constant energy sections at low binding energies correspond to sections of the repeated Brillouin zone scheme close to the $\Gamma-\mathrm{X}-\Sigma$ plane. Fig. 3(e) illustrates the actual spherical section. This measurement averages over many domains with the Néel vector pointing along all four possible $\langle 110\rangle$ directions. Correspondingly, the constant energy section is expected to display a four-fold symmetry. Sections shown in Figs. 3(a-d) are symmetrized, accordingly.

Figs. 3(c,d) show results obtained for excitation with $6.4 \mathrm{eV}$ photons using ToFMM for comparison. For this measurement, the photoemission intensity stems from a region of interest of $4.5 \mu \mathrm{m}$ diameter on the sample surface, which still averages over several domains [see Fig. 2(b,c)]. Here, the lower photon energy leads to a maximum perpendicular momentum at $k_{x}=k_{y}=0$ of $k_{z}=2.07 \AA^{-1}=1.4 G_{001}$. Thus, the probed section in the repeated Brillouin zone scheme is now close to the $\mathrm{Z}-\Sigma$-Y plane, as sketched in Fig. 3(e). The lower photon energy restricts the observable parallel momentum to $k_{\|}<0.6 \AA^{-1}$, indicating the photoemission horizon. Using the time-of-flight detection, we simultaneously acquire a data array $I\left(E_{B}, k_{x}, k_{y}\right)$ where constant energy sections can, during post-processing, be selected for different binding energies.

For $E_{B}=0.18 \mathrm{eV}$, we find the features B in Fig. 3(b), which originate from the same band as observed for $21.2 \mathrm{eV}$ in Fig. 3(a) closer to the $\mathrm{Z}$ point. At the higher binding energy $E_{B}=0.4 \mathrm{eV}$ this band seemingly broadens [Fig. 3(b)] and leads to the circular-shaped high intensity for $6.4 \mathrm{eV}$ excitation in Fig. 3(d) near the photoemission horizon. These results confirm that $I\left(E_{B}, k_{x}, k_{y}\right)$ probes the spectral function (modulated by photoemission transition probabilities) also at this low photon en- ergy, despite the fact that the final state is less freeelectron like, which leads to an integration over a finite $k_{z}$-interval.

Next, we inserted a $10 \mu \mathrm{m}$ field aperture, mounted on a piezo-adjustable holder, at the position of a Gaussian image. The latter is magnified by a factor of 11 at this position. Thus, the field aperture restricts electron paths to a circular shaped region of interest with a diameter of $0.9 \mu \mathrm{m}$. The electron optics downstream from the field aperture is then switched to momentum mode such that only electrons stemming from the selected region of interest contribute to the momentum image.

Similar data arrays $I_{i}\left(E_{B}, k_{x}, k_{y}\right)$ were measured for the five positions (1-5) of the field aperture indicated in Fig. 2(c). These positions have been chosen to cover the two (red and blue) antiferromagnetic domains shown in Fig. 2(c). To reveal the asymmetry in the intensity distribution in momentum space arising from the domains in the aperture in the spatial position $i$ we rescale the intensity signal according to

$$
\tilde{I}_{i}\left(E_{B}, k_{x}, k_{y}\right)=\frac{I_{i}\left(E_{B}, k_{x}, k_{y}\right)-\overline{I\left(E_{B}, k_{x}, k_{y}\right)}}{\overline{I\left(E_{B}, k_{x}, k_{y}\right)}},
$$

where the mean intensity distribution in momentum space is given by $\overline{I\left(E_{B}, k_{x}, k_{y}\right)}=\sum_{i} I_{i}\left(E_{B}, k_{x}, k_{y}\right) / N$. In the absence of magnetic order $\tilde{I}_{i}$ will be zero. Fig. 3 (g1) depicts this asymmetry distribution for position 1 at a binding energy $E_{B}=0.4 \mathrm{eV}$, revealing a left/right asymmetry. To determine its dependence on $E_{B}$ the asymmetry is averaged over the indicated left and right circular areas, $\tilde{I}_{l}\left(E_{B}\right)$ and $\tilde{I}_{r}\left(E_{B}\right)$. We then define the APV as $\mathrm{A}_{\mathrm{APV}} \equiv \tilde{I}_{l}\left(E_{B}\right)-\tilde{I}_{r}\left(E_{B}\right)$, depicted in Fig. 3(f) by open blue squares. We find a broad maximum value of $A_{\mathrm{APV}}=0.03$ between $E_{B}=0.2 \mathrm{eV}$ and $0.6 \mathrm{eV}$. This is consistent with the DFT bandstructure calculations shown above panel (f) at energies $0.0,0.2$, and $0.4 \mathrm{eV}$. At $0.0 \mathrm{eV}$ energy there is no anisotropy.

At position 1 the magnetic linear dichroism observed in PEEM mode [Fig. 2(c)] indicates a Néel vector alignment parallel to the $y$-axis. According to the theoretical prediction this should lead to an $\operatorname{APV}(E(k) \neq E(-k))$ perpendicular to the Néel vector, i.e. along the $x$-axis, in agreement with the experimental observation. At positions 2 and 3 the APV is also oriented along the $x$-axis but reversed [Fig. 3(g-2,g-3)]. The reversed APV thus indicates antiparallel Néel vectors in regions 1 as compared to 2 and 3 . Positions 4 and 5 show an asymmetry along the $y$-axis [Fig. 3(g-4,g-5)], revealing a Néel vector orientation parallel to the $x$-axis. This is in agreement with the magnetic linear dichroism observed in the spatial domain distribution, too, where these regions appear blue in Fig. 2(c). The reversed asymmetry between region 4 and region 5 indicates an antiparallel orientation of the Néel vector in these two domains. The asymmetry distributions observed for the five regions of interest defined 

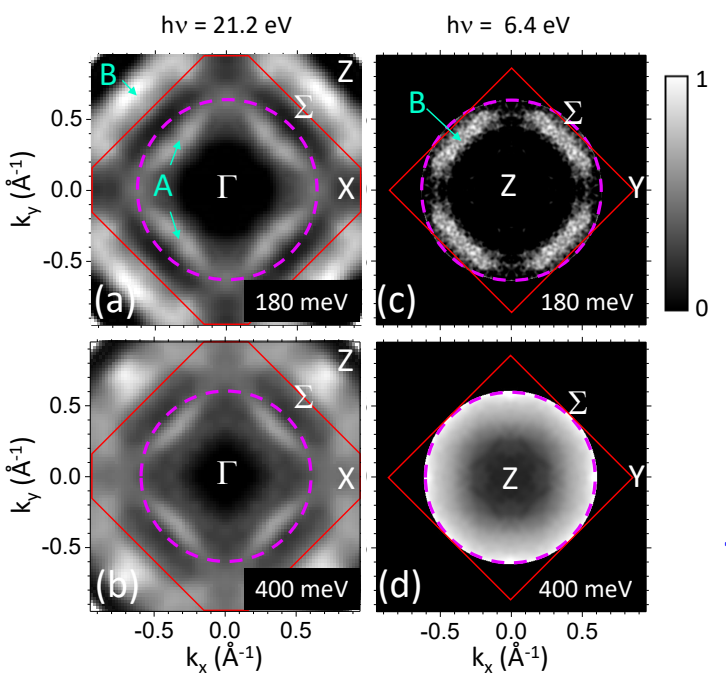

(e)
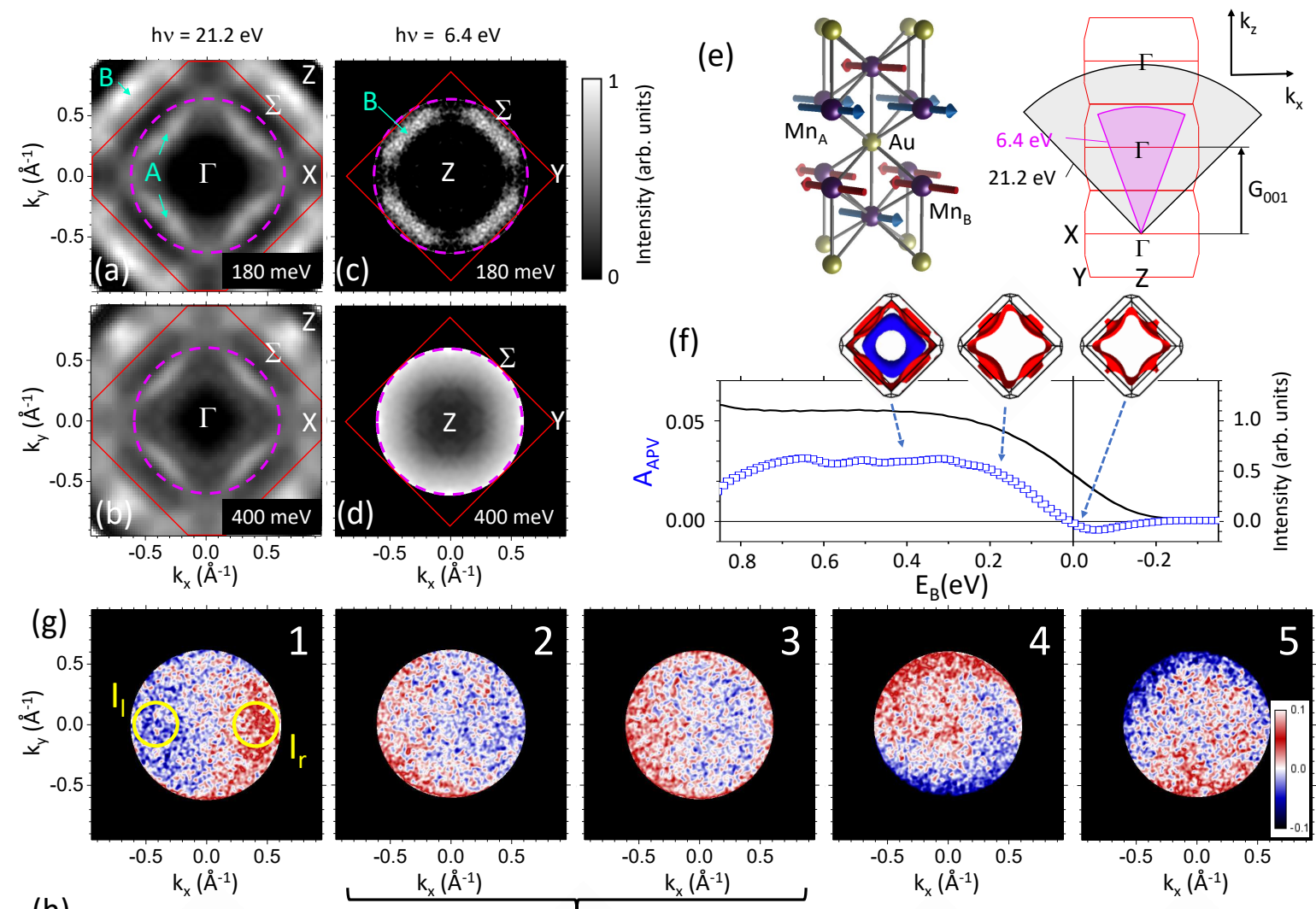

(h)

$\mathrm{h}$
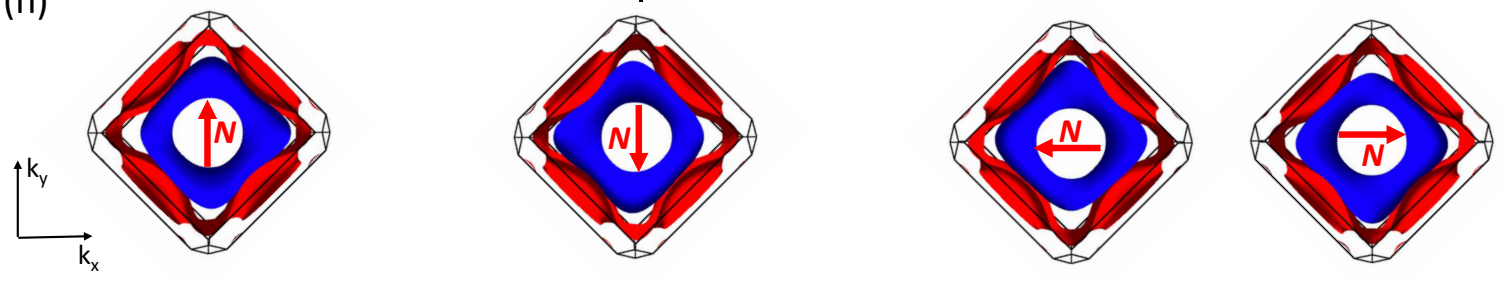

FIG. 3. (a,b) Momentum imaging with $21.2 \mathrm{eV}$ photon energy, averaged over many domains. Constant energy section at a binding energy of (a) $E_{B}=0.18 \mathrm{eV}$ and (b) $E_{B}=0.4 \mathrm{eV}$ of the photoemission intensity $I\left(E_{B}, k_{x}, k_{y}\right)$, corresponding to a cut through the constant energy surface near the $\Gamma-\mathrm{X}-\Sigma$ plane as indicated in (e). (c,d) Similar data for excitation with $6.4 \mathrm{eV}$. In this case, the momentum images correspond to cuts through the constant energy surfaces near the Z-Y- $\Sigma$ plane (c,e). Red lines indicate the corresponding Brillouin zone boundaries. The low photon energy (6.4 eV) restricts the field-of-view in momentum space to the violet dashed-circle (photoemission horizon) that is shown also in (a,b) for comparison. (e) Schematic representation of crystal lattice and repeated Brillouin zone scheme in the $k_{z}-k_{x}$ plane, indicating the $k_{z}$ values and observable $k_{\|}$range for $21.2 \mathrm{eV}$ and $6.4 \mathrm{eV}$ excitation (spherical surface section appearing circular in top view). (f) Photoemission intensity (black line) and $A_{\mathrm{APV}}$ (squares, see text for definition) for $6.4 \mathrm{eV}$ excitation versus binding energy measured at the spatial region 1 in Fig. 2(c). Also shown above the graph are the calculated bandstructures at binding energy $0.0,0.2$, and 0.4 eV, as in Fig. 1(b). (g) Antiferromagnetic parity violation measured at a binding energy of $0.4 \mathrm{eV}$ for the five regions $1-5$ defined in Fig. 2(c). The corresponding asymmetry scale is indicated by the color scale bar in the inset of (g-5). The yellow circles in (g-1) define the momentum areas used to determine the asymmetry values versus binding energy shown in (f). (h) Calculated constant energy surfaces in $k$-space at $E_{B}=0.4 \mathrm{eV}$ for the indicated Néel vectors $\mathbf{N}$ (red arrows).

by the position of the field aperture thus reveal all four possible orientations of the Néel vector, an information impossible to obtain with conventional ARPES (without sub- $\mu$ m spatial resolution).

The calculated constant energy surfaces (see Methods) shown in Figs. 3(h) reveal the origin of the experimentally observed APV. The APV results in a pronounced extension of the inner toroidal (blue) surface in the direction perpendicular to the Néel vector. More specifi- cally, the extension occurs to the right with respect to the Néel vector orientation. Figs. 3(h) depict the Néel vector orientations in accordance with the observed asymmetry in each of the measured regions. The toroidal surface, completely lying inside the first Brillouin zone, is barely touched by the nominal section probed with $6.4 \mathrm{eV}$ excitation [see Fig. 3(e)], explaining the absence of clear direct transition features in the intensity maps. On the other hand, low photon energies lead to a probed spher- 
ical section integrated over a more extended $k_{z}$ interval. The extension of the toroidal surfaces in Figs. 3(h) then result in increased photoemission intensities stemming from averaged direct transitions. Thus, theory provides a direct link between the experimentally observed asymmetry and the broken parity symmetry of energies.

The connection of the DFT calculations and the sub$\mu$-ToFMM technique gives us then a novel direct imaging technique that resolves the direction of the Néel vector domains, rather than only their alignment. Although similar information could in principle be obtained by the second-harmonic generation [32], this Néel vector imaging technique has proven very challenging in metallic systems, being most successful in insulating magnetoelectric materials. The method is viable in principle in many antiferromagnets described by one of the $21 \mathcal{P} \mathcal{T}$ symmetric magnetic point groups, which account for a large fraction (17\%) of all magnetic point groups[33].

In summary, we have directly observed an APV, $E(k) \neq E(-k)$, in the collinear antiferromagnet $\mathrm{Mn}_{2} \mathrm{Au}$. The APV is caused by the combined effect of the collinear magnetic structure of $\mathrm{Mn}_{2} \mathrm{Au}$, where the two magnetic sublattices are connected via inversion symmetry, lifted by the magnetic moments, and the spin-orbit coupling. In addition, we have demonstrated that this APV in combination with the sub- $\mu$-ToFMM technique, allows the identification of directional Néel vector domains.

This work was funded by the Deutsche Forschungsgemeinschaft (DFG, German Research Foundation) Grant No. TRR 173268565370 (projects A01, A02, A03, and A05), by the BMBF (projects 05K16UM1 and 05K19UM2), by the EU FET Open RIA Grant no. 766566, and by the Grant Agency of the Czech Republic grant no. 19-28375X. Sincere thanks are due to A. Oelsner (Surface Concept $\mathrm{GmbH}$ ) for continuous support.

[1] J. A. Sobota, Y. He, and Z.-X. Shen, Rev. Mod. Phys. 93, 025006 (2021).

[2] L. Šmejkal, J. Sinova, and T. Jungwirth, (2021), arXiv:2105.05820.

[3] L. Smejkal, J. Zelezny, J. Sinova, and T. Jungwirth, Phys. Rev. Lett. 118, 106402 (2017).

[4] S. Hayami, M. Yatsushiro, Y. Yanagi, and H. Kusunose, Phys. Rev. B 98, 165110 (2018).

[5] H. Watanabe and Y. Yanase, Physical Review X 11, 011001 (2021).

[6] H. J. Elmers, S. V. Chernov, S. W. DSouza, S. P. Bommanaboyena, S. Y. Bodnar, K. Medjanik, S. Babenkov, O. Fedchenko, D. Vasilyev, S. Y. Agustsson, C. Schlueter, A. Gloskovskii, Y. Matveyev, V. N. Strocov, Y. Skourski, L. Šmejkal, J. Sinova, J. Minar, M. Klui, G. Schnhense, and M. Jourdan, ACS Nano 14, 17554 (2020), pMID: 33236903, https://doi.org/10.1021/acsnano.0c08215.

[7] S. Hayami, Y. Yanagi, and H. Kusunose, Physical Review B 102, 144441 (2020).
[8] C. Carbone, P. Moras, P. M. Sheverdyaeva, D. Pacilé, M. Papagno, L. Ferrari, D. Topwal, E. Vescovo, G. Bihlmayer, F. Freimuth, Y. Mokrousov, and S. Blügel, Phys. Rev. B 93, 125409 (2016).

[9] J. Zelezny, H. Gao, K. Vyborny, J. Zemen, J. Masek, A. Manchon, J. Wunderlich, J. Sinova, and T. Jungwirth, Phys. Rev. Lett. 113, 157201 (2014).

[10] P. Wadley, B. Howells, J. Zelezny, C. Andrews, V. Hills, R. P. Campion, V. Novak, K. Olejnik, F. Maccherozzi, S. S. Dhesi, S. Y. Martin, T. Wagner, J. Wunderlich, F. Freimuth, Y. Mokrousov, J. Kunes, J. S. Chauhan, M. J. Grzybowski, A. W. Rushforth, and K. W. et al. Edmonds, Science 351, 587 (2016).

[11] S. Y. Bodnar, L. Smejkal, I. Turek, T. Jungwirth, O. Gomonay, J. Sinova, A. A. Sapozhnik, H. J. Elmers, M. Kläui, and M. Jourdan, Nat. Comm. 9, 348 (2018).

[12] J. Godinho, H. Reichlova, D. Kriegner, V. Novak, K. Olejnik, Z. Kaspar, Z. Soban, P. Wadley, R. P. Campion, R. M. Otxoa, P. E. Roy, J. Zelezny, T. Jungwirth, and J. Wunderlich, Nat. Commun. 9, 4686 (2018), arXiv:1806.02795.

[13] S. Bommanaboyena, B. Backes, L. S. I. Veiga, S. S. Dhesi, Y. R. Niu, B. Sarpi, T. Denneulin, A. Kovacs, T. Mashoff, O. Gomonay, J. Sinova, K. Everschor-Sitte, D. Schönke, R. M. Reeve, M. Kläui, H.-J. Elmers, and M. Jourdan, Arxiv (2021).

[14] M. Jourdan, H. Braeuning, A. Sapozhnik, H. J. Elmers, H. Zabel, and M. Klaeui, J. Phys. D: Appl. Phys. 48, 385001 (2015).

[15] S. P. Bohammaboyena, T. Bergfeldt, R. Heller, M. Kläui, and M. Jourdan, J. Appl. Phys. 127, 243901 (2020).

[16] A. A. Sapozhnik, M. Filianina, S. Y. Bodnar, A. Lamirand, M.-A. Mawass, Y. Skourski, H.-J. Elmers, H. Zabel, M. Klaeui, and M. Jourdan, Phys. Rev. B 97, 134429 (2018).

[17] A. Hubert and R. Schäfer, Magnetic Domains (Springer (Berlin), 1998).

[18] A. V. Kimel, G. V. Astakhov, A. Kirilyuk, G. M. Schott, G. Karczewski, W. Ossau, G. Schmidt, L. W. Molenkamp, and T. Rasing, Phys. Rev. Lett. 94, 227203 (2005).

[19] G. K. L. Marx, H. J. Elmers, and G. Schönhense, Phys. Rev. Lett. 84, 5888 (2000).

[20] T. Nakagawa and T. Yokoyama, Phys. Rev. Lett. 96, 237402 (2006).

[21] K. Hild, J. Maul, G. Schönhense, H. J. Elmers, M. Amft, and P. M. Oppeneer, Phys. Rev. Lett. 102, 057207 (2009).

[22] T. Nakagawa and T. Yokoyama, J. Electr. Spectr. Rel. Phenom. 185, 356 (2012).

[23] F. U. Hillebrecht, T. Kinoshita, D. Spanke, J. Dresselhaus, C. Roth, H. B. Rose, and E. Kisker, Phys. Rev. Lett. 75, 2224 (1995).

[24] J. Stohr, A. Scholl, T. Regan, S. Anders, J. Luning, M. Scheinfein, H. Padmore, and R. White, Phys. Rev. Lett. 83, 1862 (1999).

[25] F. Nolting, A. Scholl, J. Stohr, J. Seo, J. Fompeyrine, H. Siegwart, J. Locquet, S. Anders, J. Luning, E. Fullerton, M. Toney, M. Scheinfein, and H. Padmore, Nature 405, 767 (2000).

[26] I. P. Krug, F. U. Hillebrecht, M. W. Haverkort, A. Tanaka, L. H. Tjeng, H. Gomonay, A. FraileRodrifguez, F. Nolting, S. Cramm, and C. M. Schneider, Phys. Rev. B 78, 064427 (2008). 
[27] L. Baldrati, O. Gomonay, A. Ross, M. Filianina, R. Lebrun, R. Ramos, C. Leveille, F. Fuhrmann, T. R. Forrest, F. Maccherozzi, S. Valencia, F. Kronast, E. Saitoh, J. Sinova, and M. Klaeui, Phys. Rev. Lett. 123, 177201 (2019).

[28] P. Wadley, S. Reimers, M. J. Grzybowski, C. Andrews, M. Wang, J. S. Chauhan, B. L. Gallagher, R. P. Campion, K. W. Edmonds, S. S. Dhesi, F. Maccherozzi, V. Novak, J. Wunderlich, and T. Jungwirth, Nat. Nanotech. 13, 362 (2018).

[29] V. Grigorev, M. Filianina, S. Y. Bodnar, S. Sobolev, N. Bhattacharjee, S. Bommanaboyena, Y. Lytvynenko, Y. Skourski, D. Fuchs, M. Kläui, M. Jourdan, and J. Demsar, Phys. Rev. Applied 16, 014037 (2021).

[30] S. Hüfner, Photoelectron Spectroscopy - Principles and Applications (Springer, Berlin, 2003).

[31] F. Reinert and S. Hüfner, New J. Phys. 7, 97 (2005).

[32] M. Fiebig, D. Frohlich, B. B. Krichevtsov, and R. V. Pisarev, Phys. Rev. Lett. 73, 2127 (1994).

[33] J. Schütte-Engel, D. J. Marsh, A. J. Millar, A. Sekine, F. Chadha-Day, S. Hoof, M. N. Ali, K. C. Fong, E. Hardy, and L. Šmejkal, Journal of Cosmology and Astroparticle Physics 2021, 066 (2021).

[34] G. Schönhense, S. Babenkov, D. Vasilyev, H.-J. Elmers, and K. Medjanik, Rev. Sci. Instr. 91, 123110 (2020), https://doi.org/10.1063/5.0024074.

[35] K. Dewhurst, "Elk code," (2021).

[36] M. Kawamura, Computer Physics Communications 239, 197 (2019).

\section{SUPPLEMENTARY MATERIAL AND METHODS}

\section{Experimental Details}

Samples were transported from the deposition chamber to the photoemission experiment using an ultra-high vacuum suitcase. For ARPES measurements, photoelectrons were excited by a He discharge lamp $(21.2 \mathrm{eV})$ and by a pulsed laser $(6.4 \mathrm{eV}, 80 \mathrm{MHz}$ repetition rate, APE). The incidence angle of the photon beam is $22^{0}$ with respect to the sample surface along the $x$-axis. The samples have been aligned such that the $x$ and $y$ directions correspond to the magnetic $\langle 110\rangle$ easy axes, respectively. Photoemission experiments at $21.2 \mathrm{eV}$ have been performed using the single-hemisphere momentum microscope described in Ref. [34] with the energy resolution set to $50 \mathrm{meV}$ and laser ARPES experiments using a ToFMM (Surface Concept GmbH) with the resolution set to $40 \mathrm{meV}$. For the latter experiment, a field aperture inserted at the position of a Gaussian image allows to restrict the region of interest to a circular area with $0.9 \mu \mathrm{m}$ diameter, while the downstream electron optics can be set from Gaussian to Fourier imaging for momentummapping.

\section{Theoretical Methods}

For the equilibrium density functional theory calculations and symmetry analysis, we used the FLAPW code ELK [35]. We used the BCT unit cell and a k-point mesh $10 \times 10 \times 10$. We plot the Fermi surfaces with the program Fermisurfer [36]. More details are given in Refs. 6 and 11. 